\title{
MAIN-SEQUENCE STAR POPULATIONS IN THE VIRGO OVERDENSITY REGION*
}

\author{
H. Jerjen ${ }^{1}$, G. S. Da Costa ${ }^{1}$, B. Willman ${ }^{2}$, P. Tisserand ${ }^{1}$, N. Arimoto ${ }^{3,4}$, S. Okamoto ${ }^{5}$, M. Mateo ${ }^{6}$, I. Saviane ${ }^{7}$,

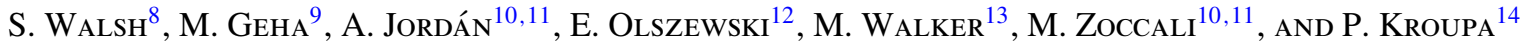 \\ ${ }^{1}$ Research School of Astronomy \& Astrophysics, The Australian National University, Mt Stromlo Observatory, via Cotter Road, Weston, ACT 2611, Australia \\ ${ }^{2}$ Haverford College, Department of Astronomy, 370 Lancaster Avenue, Haverford, PA 19041, USA \\ ${ }^{3}$ National Astronomical Observatory of Japan, Subaru Telescope, 650 North A'ohoku Place, Hilo, HI 96720, USA \\ ${ }^{4}$ The Graduate University for Advanced Studies, Department of Astronomical Sciences, Osawa 2-21-1, Mitaka, Tokyo, Japan \\ ${ }^{5}$ Kavli Institute for Astronomy and Astrophysics, Peking University, Beijing 100871, China \\ ${ }^{6}$ Department of Astronomy, University of Michigan, Ann Arbor, MI, USA \\ ${ }^{7}$ European Southern Observatory, Casilla 19001, Santiago 19, Chile \\ ${ }^{8}$ Australian Astronomical Observatory, PO Box 915, North Ryde, NSW 1670, Australia \\ ${ }_{9}^{9}$ Astronomy Department, Yale University, New Haven, CT 06520, USA \\ ${ }^{10}$ Departamento de Astronomía y Astrofísica, Pontificia Universidad Católica de Chile, 7820436 Macul, Santiago, Chile \\ ${ }^{11}$ The Milky Way Millennium Nucleus, Av. Vicuña Mackenna 4860, 782-0436 Macul, Santiago, Chile \\ 12 Steward Observatory, The University of Arizona, Tucson, AZ, USA \\ ${ }^{13}$ Harvard-Smithsonian Center for Astrophysics, 60 Garden Street, Cambridge, MA 02138, USA \\ ${ }^{14}$ Argelander Institute for Astronomy, University of Bonn, Auf dem Hügel 71, D-53121 Bonn, Germany \\ Received 2012 February 23; accepted 2013 March 19; published 2013 April 30
}

\begin{abstract}
We present deep color-magnitude diagrams (CMDs) for two Subaru Suprime-Cam fields in the Virgo Stellar Stream (VSS)/Virgo Overdensity (VOD) and compare them to a field centered on the highest concentration of Sagittarius (Sgr) Tidal Stream stars in the leading arm, Branch A of the bifurcation. A prominent population of main-sequence stars is detected in all three fields and can be traced as faint as $g \approx 24$ mag. Using theoretical isochrone fitting, we derive an age of $9.1_{-1.1}^{+1.0} \mathrm{Gyr}$, a median abundance of $[\mathrm{Fe} / \mathrm{H}]=-0.70_{-0.20}^{+0.15}$ dex, and a heliocentric distance of $30.9 \pm 3.0 \mathrm{kpc}$ for the main sequence of the Sgr Stream Branch A. The dominant main-sequence populations in the two VSS/VOD fields $\left(\Lambda_{\odot} \approx 265^{\circ}, B_{\odot} \approx 13^{\circ}\right)$ are located at a mean distance of $23.3 \pm 1.6 \mathrm{kpc}$ and have an age of $\sim 8.2 \mathrm{Gyr}$, and an abundance of $[\mathrm{Fe} / \mathrm{H}]=-0.67_{-0.12}^{+0.16}$ dex, similar to the Sgr Stream stars. These statistically robust parameters, derived from the photometry of 260 main-sequence stars, are also in good agreement with the age of the main population in the Sgr dwarf galaxy (8.0 $\pm 1.5 \mathrm{Gyr})$. They also agree with the peak in the metallicity distribution of $2-3$ Gyr old M giants, $[\mathrm{Fe} / \mathrm{H}] \approx-0.6 \mathrm{dex}$, in the Sgr north leading arm. We then compare the results from the VSS/VOD fields with the Sgr Tidal Stream model by Law \& Majewski based on a triaxial Galactic halo shape that is empirically calibrated with Sloan Digital Sky Survey Sgr A-branch and Two Micron All Sky Survey M-giant stars. We find that the most prominent feature in the CMDs, the main-sequence population at $23 \mathrm{kpc}$, is not explained by the model. Instead the model predicts in these directions a low-density filamentary structure of Sgr debris stars at $\sim 9 \mathrm{kpc}$ and a slightly higher concentration of Sgr stars spread over a heliocentric distance range of $42-53 \mathrm{kpc}$. At best there is only marginal evidence for the presence of these populations in our data. Our findings then suggest that while there are probably some Sgr debris stars present, the dominant stellar population in the VOD originates from a different halo structure that has an almost identical age and metallicity as some sections of the Sgr tidal stream.
\end{abstract}

Key words: galaxies: individual (Sagittarius) - galaxies: stellar content - Galaxy: abundances - Galaxy: halo - Galaxy: structure

\section{INTRODUCTION}

The first decade of wide-field digital imaging has revolutionized the way the three-dimensional (3D) structure of the Milky Way can be mapped. The tomographic studies enabled by programs such as the Sloan Digital Sky Survey (SDSS; York et al. 2000) or the Two Micron All Sky Survey (2MASS; Skrutskie et al. 2006) have not only uncovered members from a new family of ultra-faint satellite galaxies but also revealed strong evidence for the presence of a significant amount of substructure in the halo of the Milky Way in the form of largescale stellar streams. Understanding the nature and origin of these satellite galaxies and their tidal debris has major implications for the validity of cosmological models (e.g., Kroupa et al. 2010). The most striking satellite galaxy currently undergoing

\footnotetext{
* Based on data collected at Subaru Telescope, which is operated by the National Astronomical Observatory of Japan.
}

tidal disruption is the Sagittarius (Sgr) dwarf (Ibata et al. 1994, 2001; Martínez-Delgado et al. 2001; Majewski et al. 2003). This galaxy is located approximately $16 \mathrm{kpc}$ from the Galactic center (Kunder \& Chaboyer 2009), but the debris from the interaction with the Milky Way have been traced from 16-90 kpc galactocentric distance across large areas of the sky (Majewski et al. 2003; Newberg et al. 2003; Belokurov et al. 2006; Correnti et al. 2010). Layden \& Sarajedini (2000) found evidence for multiple epochs of star formation in the Sgr dwarf with the principal star formation epochs at 11,5 , and $0.5-3 \mathrm{Gyr}$ and associated mean abundance values of $[\mathrm{Fe} / \mathrm{H}]=-1.3,-0.7$, and -0.4 , respectively. This picture was subsequently refined by Bellazzini et al. (2006), who found that more than $80 \%$ of Sgr stars belong to a relatively metal-rich $([\mathrm{Fe} / \mathrm{H}] \sim-0.7 \mathrm{dex})$ and intermediate-old age $(8.0 \pm 1.5 \mathrm{Gyr})$ population called Pop A, after Bellazzini et al. (1999) and Monaco et al. (2002).

Besides major tidal streams, the analysis of large samples of tracers of the halo substructure has further revealed the presence 
of numerous smaller stellar overdensities possibly associated with unknown fainter streams or merger events in the halo. In particular, for the region of the Virgo constellation, several detections of stellar overdensities have been reported covering the distance range 4-20 kpc. Most noticeable is a significant concentration of RR Lyrae (RRL) stars at $\alpha=12.4 \mathrm{~h}$ (Vivas et al. 2001; Vivas 2002; Vivas \& Zinn 2003; Zinn et al. 2004; Ivezić et al. 2005) located at $\approx 19 \mathrm{kpc}$ from the Sun (Duffau et al. 2006; Newberg et al. 2007; Prior et al. 2009b). This clump was also detected as an excess of metal-poor, old F-type main-sequence (MS) turnoff stars by Newberg et al. (2002) and as a distinct stellar clustering labeled Vir Z by Walsh et al. (2009). Duffau et al. (2006) showed via spectroscopy that the majority of the RRL and blue horizontal branch stars in the overdense region define a kinematically cold $\left(\sigma \approx 20 \mathrm{~km} \mathrm{~s}^{-1}\right)$ feature centered on a Galactic rest-frame velocity $V_{\mathrm{GSR}}$ of $\sim 100 \mathrm{~km} \mathrm{~s}^{-1}$, which they called the "Virgo Stellar Stream" (VSS).

Furthermore, Jurić et al. (2008) and more recently Bonaca et al. (2012) detected a density enhancement over $2000 \mathrm{deg}^{2}$ of sky toward the Virgo constellation by means of photometric parallax distance estimates of SDSS stars. They called this large-scale feature the "Virgo Overdensity" (VOD). The VOD is estimated to have a distance of 6-20 kpc (Jurić et al. 2008; Vivas et al. 2008; Keller et al. 2009). In the following, we will use the term "VSS" when we are referring to the feature identified kinematically and abundance-wise, and use the term "VOD" for the spatial overdensity.

Martínez-Delgado et al. (2004, 2007) speculated that the Virgo stellar overdensity might be related to the complex debris structure of the trailing arm of the Sgr stream, a scenario that is supported by the Sgr Stream models for this region of the sky (Law et al. 2005; Fellhauer et al. 2006) assuming that the Galactic halo potential has a spherical or oblate shape. Subsequent spectroscopic follow-up studies of a sample of these RRLs by Prior et al. (2009b) not only found further RRLs associated with the VSS, both in terms of velocity and abundance, but also revealed a population of metal-poor RRLs with large negative $V_{\mathrm{GSR}}$ velocities. These stars were taken to indicate the likely presence of a population of Sgr leading tidal tail stars, which are expected to have such velocities in this region. Prior et al. (2009a) also suggested that Sgr trailing debris may make a contribution to the population at positive $V_{\mathrm{GSR}}$ velocities, though it was unlikely to fully account for the VSS feature. Chou et al. $(2007,2010)$ have argued from a chemical abundance point of view for the presence of Sgr debris stars in this region.

The aim of the present paper is to provide a robust estimate of the mean age of the stellar population that dominates the VSS/VOD region by means of deep imaging in two directions close to the $12.4 \mathrm{~h}$ clump. For that purpose we observed two Subaru Suprime-Cam fields covering $0.25 \mathrm{deg}^{2}$ each, centered around $\alpha_{2000}=12^{\mathrm{h}} 20^{\mathrm{m}} 18^{\mathrm{s}}, \delta_{2000}=-01^{\mathrm{d}} 21^{\mathrm{m}} 00^{\mathrm{s}}$ and $\alpha_{2000}=12^{\mathrm{h}} 47^{\mathrm{m}} 58^{\mathrm{s}}, \delta_{2000}=-00^{\mathrm{d}} 45^{\mathrm{m}} 00^{\mathrm{s}}$, respectively. We also observed a field $19^{\circ}$ away, in the direction of the highest star density of the Sgr Branch A leading arm. All three fields were identified by Walsh et al. (2009) as having statistically significant overdensities of point sources.

In Section 2, we describe the data acquisition, reduction, and photometric calibration. The analysis of the color-magnitude diagrams (CMDs) is presented in Section 3. In Section 4, arguments are compiled for the interpretation of our measurements in the context of the VOD being dominated by the Sgr Tidal Stream. An alternative interpretation of the CMDs naturally arises from the comparison with the predictions of the
Table 1

Coordinates of the Sgr Tidal Stream and the Two Virgo Fields

\begin{tabular}{lcccccr}
\hline \hline Field & $\begin{array}{c}\alpha_{2000} \\
(\mathrm{deg})\end{array}$ & $\begin{array}{c}\delta_{2000} \\
(\mathrm{deg})\end{array}$ & $\begin{array}{c}l \\
(\mathrm{deg})\end{array}$ & $\begin{array}{c}b \\
(\mathrm{deg})\end{array}$ & $\begin{array}{c}\Lambda_{\odot} \\
(\mathrm{deg})\end{array}$ & $\begin{array}{c}B_{\odot} \\
(\mathrm{deg})\end{array}$ \\
\hline $1145+13$ & 176.288 & +13.95 & 250.07 & +69.68 & 247.8 & 5.2 \\
$1220-01$ & 185.077 & -01.35 & 286.95 & +60.55 & 262.1 & 15.3 \\
$1247-00$ & 191.992 & -00.75 & 301.07 & +62.11 & 268.6 & 11.4 \\
\hline
\end{tabular}

Sgr dwarf-Milky Way halo interaction model. This is tested in Section 5. Finally, in Section 6 we summarize and discuss our conclusions.

\section{DATA ACQUISITION, PHOTOMETRY, AND CALIBRATION}

We obtained deep $g, r$ CCD images of the three fields (Table 1) using the Suprime-Cam (Miyazaki et al. 2002) on the Subaru Telescope during nights of 2009 February 22 and 23 (PI: N. Arimoto). The Suprime-Cam consists of a $5 \times 2$ array of $2048 \times 4096$ CCD detectors and provides a field of view (FoV) of $34^{\prime} \times 27^{\prime}$ with a pixel scale of $0^{\prime} .202$. The first night was photometric and the second night was partially clear with the median seeing during both nights at 0 '.8. Each field was observed in a dithered series of $5 \times 280 \mathrm{~s}$ exposures in the SDSS $g$ band and $10 \times 200 \mathrm{~s}$ in $r$ band. Data were processed using the pipeline software SDFRED dedicated to the Suprime-Cam (Yagi et al. 2002; Ouchi et al. 2004). Each image was bias-subtracted and trimmed, flat-fielded, distortion and atmospheric dispersion corrected, sky-subtracted, and combined in the usual manner. The astrometric calibration of each passband was based on a general zenithal polynomial projection derived from astrometric standard stars selected from the online USNO catalog. ${ }^{15}$

SourceExtractor (Bertin \& Arnouts 1996) was employed to identify and analyze sources down to $g \sim 26 \mathrm{mag}$. The source catalogs include point-spread function (PSF) and flux measurements inside two circular apertures (diameter: 0.'8 and $1^{\prime \prime} .2$ ) optimized for the seeing. We tied our photometry to the SDSS photometric system by matching all the point sources from SDSS observed in our fields with our $g$-band and $r$-band detections. The magnitude zero points with respect to the Galactic-extinction-corrected SDSS photometry were obtained by calculating a $3 \sigma$ clipped mean of the magnitude differences of the unsaturated SDSS stars that were detected in our Subaru data. The comparison of typically 100-200 SDSS stars in the magnitude interval $17.6<g, r<22.5$ for each calibration made these zero points statistically robust with uncertainties less than $0.01 \mathrm{mag}$.

To separate stars from background galaxies and other nonstellar objects, we compare the fluxes in the two different apertures. The PSF geometry places stellar objects in a welldefined, narrow region in the magnitude-flux ratio plane. Such a plot is shown in Figure 1 for the 1220-01 field. Unsaturated stars populate vertical bands centered at $g_{0.8}-g_{1.2}=0.52$ and $r_{0.8}-r_{1.2}=0.54$, respectively. Selecting only objects with values in these two bands ensures a fair star-galaxy separation in our images down to $g \sim 23.5-24.0 \mathrm{mag}$, a limit that depends on the seeing. At fainter magnitudes the signal to noise gets too low and unresolved background galaxies blend into the region leading to an increasing level of contamination. All CMDs $\left((g-r)_{0}\right.$ versus $\left.g_{0}\right)$ shown in our study are based on aperture

\footnotetext{
15 http://ftp.nofs.navy.mil/data/fchpix/
} 

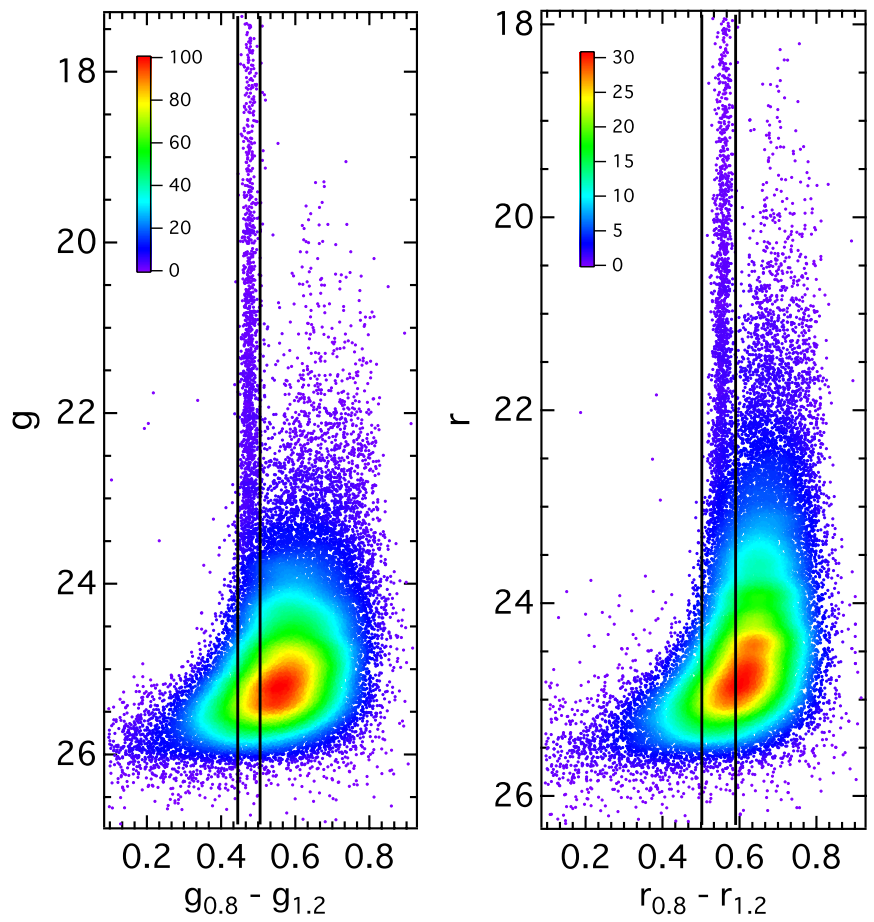

Figure 1. Example of star-galaxy separation: the aperture flux ratio vs. total flux for all detected sources in the $1220-01$ field. Unsaturated stars populate welldefined vertical bands $0.45<g_{0.8}-g_{1.2}<0.51$ and $0.52<r_{0.8}-r_{1.2}<0.59$, respectively. Selecting objects in these two narrow regions enables a fair star-galaxy separation down to $g \sim 23.7 \mathrm{mag}$. Fainter objects can be stars or unresolved background galaxies (see Figure 2). Sources are color-coded with the local number density.

photometry with a $0 . \prime 8$ diameter aperture calibrated to the SDSS system and are restricted to objects that fall simultaneously into both stellar flux ratio intervals as determined for each field individually.

Artificial star tests were conducted to estimate the detection completeness. A catalog of uniformly distributed simulated stars was added to each image based on measured PSF parameters and noise properties. Photometry of all the stars was then performed and typical photometric errors were derived as the difference between input and computed magnitude. We calculated the fraction of simulated stars recovered at different locations across the image to test whether stellar crowding is present and how it affects the completeness of the observations. The derived completeness curves (recovery rates as a function of magnitude) did not vary across the FoV and showed a steep gradient where the $50 \%$ source completeness in the photometry is approximately 0.5 mag brighter than the limiting magnitude of $g_{\text {lim }} \sim 26$ mag.

\section{COLOR-MAGNITUDE DIAGRAMS}

Over the observed FoV of $918 \operatorname{arcmin}^{2}$, several thousand sources satisfy the aperture flux selection. The three CMDs (Figure 2) show two distinct vertical plumes of Galactic stars reflecting the separation of the halo and thick disk stars at a color of $(g-r)_{0} \sim 0.3$ and thin disk stars at a color of $(g-r)_{0} \sim 1.35$ (see Figure 2(a) or Chen et al. 2001). Below $g_{0}=24 \mathrm{mag}$ and around $-0.2<(g-r)_{0}<0.8$, an increasing number of unresolved background galaxies begin to dominate the CMDs. The Subaru photometry typically reaches down to a limiting magnitude of $g_{\text {lim }} \sim 26 \mathrm{mag}$, but we will restrict our analysis to the interval $18<g<24$.
Table 2

Star Counts in the Box $1.2<g-r<1.8,20<g<24$

\begin{tabular}{lcc}
\hline \hline Field & Observed & TRILEGAL \\
\hline $1145+13$ & 367 & 395 \\
$1220-01$ & 536 & 510 \\
$1247-00$ & 546 & 568 \\
\hline
\end{tabular}

The CMDs were statistically decontaminated from unwanted Galactic stars using model CMDs generated for each field with the TRILEGAL code (Girardi et al. 2005; Vanhollebeke et al. 2009). As an example, the simulated CMD of the Galactic field in the direction of $1145+13$ is shown in Figure 2(a). A comparison of the numbers of observed and predicted Galactic stars in the magnitude-color intervals $1.2<g-r<1.8$, $20<g<24$ finds good agreement (Table 2).

The cleaning procedure involves a process in which a point source $A$ in the CMD of $1145+13,1220-00$, or $1247-01$ is removed if there is a source $\mathrm{B}$ in the Galactic CMD that lies within the $3 \sigma$ photometry uncertainty ellipse of A. Source B is also removed from the control field catalog for the rest of the process to avoid repeated use. If more than one source is found in the ellipse, the source closest to A is discarded. The decontaminated CMDs are shown in Figure 3. We note that the stars in the simulated CMD represent a single realization of the contamination, which explains why not all Galactic stars were removed. Cleaning efficiencies of $68 \%(1145+13), 63 \%$ (1220-00), and 59\% (1247-01) were achieved in the "pure field" part of the CMD $(1<g-r<2,20<g<24)$. For the $0<g-r<1$ color interval where the star density is higher compared to a pure field, the cleaning process is expected to be more efficient because of the higher probability that an observed star matches with a star in the TRILEGAL CMD and thus is removed.

\subsection{Field $1145+13$ in the Sgr Stream Leading Arm}

The tidal stream of the Sgr dwarf galaxy in the SDSS data visibly diverges westward of $\alpha \approx 190^{\circ}$ to give a prominent bifurcation known as Branches A and B (Belokurov et al. 2006). Our field $1145+13$ is located at equatorial coordinates $\alpha_{2000}=$ $176.29, \delta_{2000}=13.95$ in the direction of the highest star density of the Sgr Branch A, as defined by Belokurov et al. (2006). In line with expectations, our CMD (Figure 2(b)) exhibits a diagonal ridge of MS stars in the interval $21<g<25.5$, a feature that is even more pronounced in the statistically decontaminated version of the CMD (Figure 3). The Sgr Stream population has a measured MSTO color of $(g-r)_{0} \sim 0.25$. We derive the age, $[\mathrm{Fe} / \mathrm{H}]$, and distance of the Sgr Stream using a maximumlikelihood method which closely follows Frayn \& Gilmore (2002) and was employed to analyze the CMD of Segue 3 (Fadely et al. 2011). The fundamental assumption is that the data are dominated by a single age/metallicity population. For the procedure, a suite of isochrones are fitted to a sample of stars, assigning to each a bivariate Gaussian probability function whose variance is set by the associated photometric errors $\sigma_{g}$ and $\sigma_{(g-r)}$. We apply this analysis on all stars in our decontaminated CMD. For a given isochrone $i$, we compute the likelihood

$$
\mathcal{L}_{i}=\prod_{j} p\left(\{g, g-r\}_{j} \mid i,\{g, g-r\}_{i j}, \mathrm{DM}_{i}\right)
$$

where $p$ is defined as 

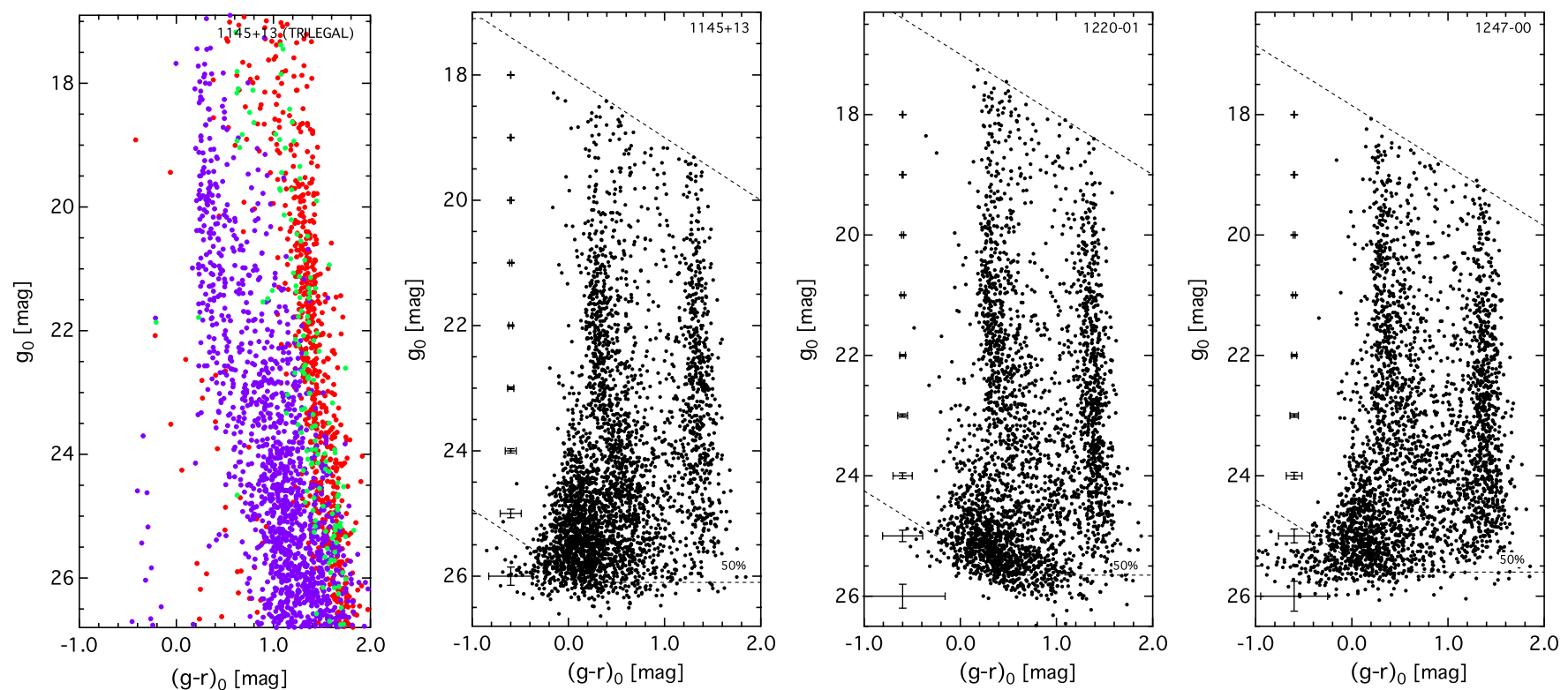

Figure 2. (a) Color-magnitude diagram of the Galactic field in the direction of $1145+13$ simulated with the TRILEGAL 1.5 code. Different stellar populations are coded in color (thin disk: red; thick disk: green; halo: blue). (b-d) Reddening-corrected color-magnitude diagrams of the three Subaru fields with representative photometric errors plotted on the left side of the CMD. (b) CMD of the field 1145+13 in the direction of the Sagittarius Stream A branch. A prominent MS population is visible with the turnoff at $g \sim 21.0$ mag. (c) CMDs of the field 1220-01 and (d) 1247-00, respectively. Similar to the 1145+13 field, a prominent MS population is visible with the turnoff at $g \sim 20.0-20.5$ mag. Unresolved background galaxies appear as plume below $g=24$ in the color interval $-0.4<g<0.6$.
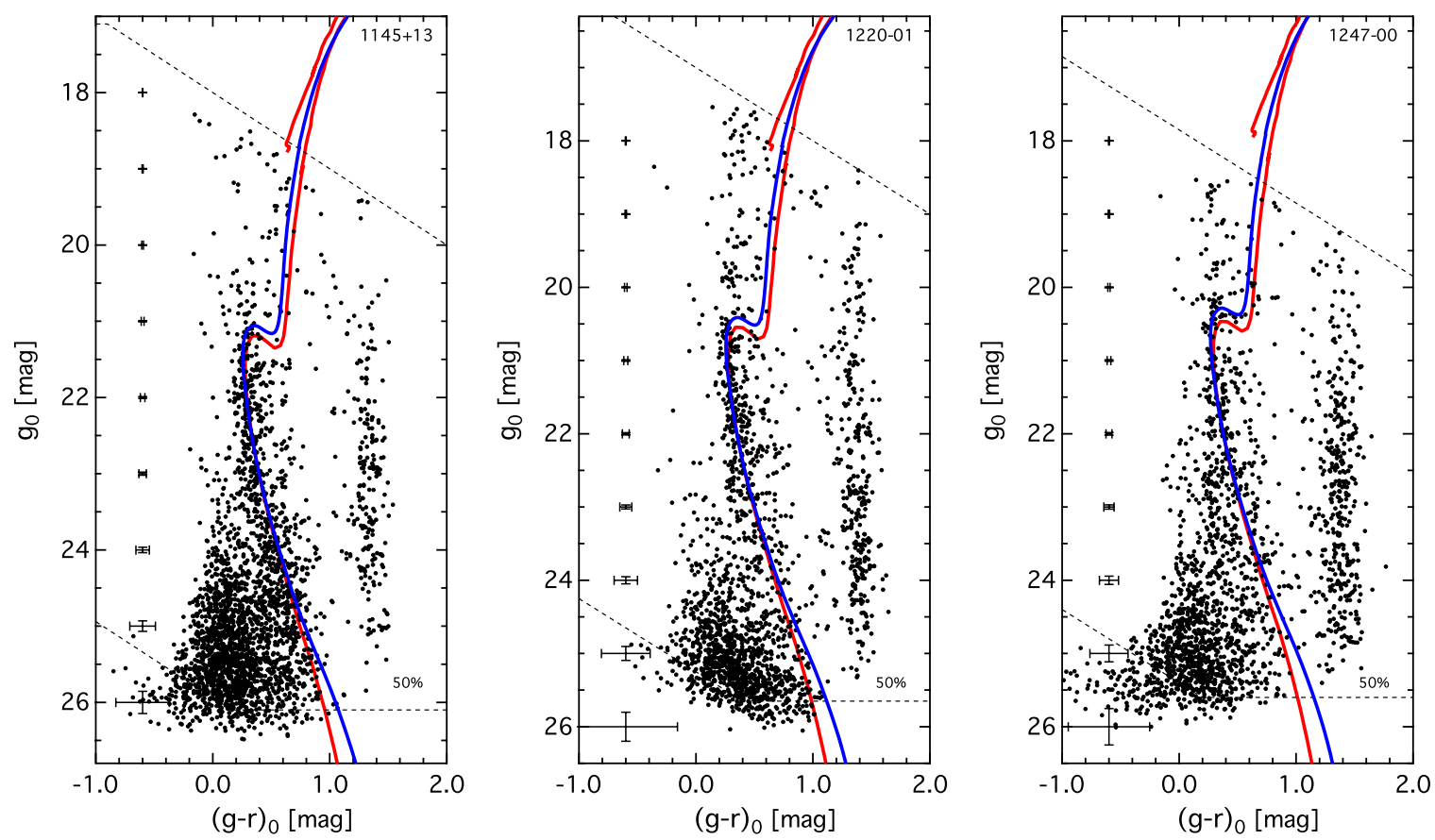

Figure 3. Foreground-subtracted CMDs with the best-fitting Dartmouth isochrones (blue line) to the main-sequence population (a) $1145+13$ has a metallicity of $[\mathrm{Fe} / \mathrm{H}]=-0.70 \mathrm{dex}$ and an age of $9.1 \mathrm{Gyr}$. The associated heliocentric distance (modulus) is $30.9 \mathrm{kpc}(m-M=17.45 \mathrm{mag}$ ). (b) $1220-01 \mathrm{kas}$ a metallicity of $[\mathrm{Fe} / \mathrm{H}]=-0.66 \mathrm{dex}$ and an age of $7.9 \mathrm{Gyr}$. The associated heliocentric distance (modulus) is $24.3 \mathrm{kpc}(m-M=16.93 \mathrm{mag}$ ). (c) $1247-00 \mathrm{has}$ a metallicity of $[\mathrm{Fe} / \mathrm{H}]=-0.68 \mathrm{dex}$ and an age of $8.5 \mathrm{Gyr}$. The associated heliocentric distance (modulus) is $22.2 \mathrm{kpc}(m-M=16.73$ mag). The Padova isochrones with the same ages, metallicities, and distances are shown in red for comparison.

$$
\begin{aligned}
& p\left(\{g, g-r\}_{j} \mid i,\{g, g-r\}_{i j}, \mathrm{DM}_{i}\right)=\frac{1}{2 \pi \sigma_{g_{j}} \sigma_{(g-r)_{j}}} \\
& \quad \times \exp \left(-\frac{1}{2}\left[\left(\frac{g_{j}-\left(g_{i j}+\mathrm{DM}_{i}\right)}{\sigma_{g_{j}}}\right)^{2}\right.\right. \\
& \left.\left.\quad+\left(\frac{(g-r)_{j}-(g-r)_{i j}}{\sigma_{(g-r)_{j}}}\right)^{2}\right]\right) .
\end{aligned}
$$

For each star, $j,\{g, g-r\}_{i j}$, and $\mathrm{DM}_{i}$ are the magnitude, color, and de-reddened distance modulus values for isochrone $i$ that maximize the likelihood of the entire data set $\{g, g-r\}_{j}$ in Equation (2). We take an approximate solution to finding the values of $\{g, g-r\}_{i j}$ and $\mathrm{DM}_{\mathrm{i}}$ by searching over a series of fine steps in $g, g-r$, and DM values for each isochrone. Input isochrones are supplied by the Dartmouth library (Dotter et al. 2008), and linearly interpolated at a step size of $0.01 \mathrm{mag}$ in the two-dimensional color-magnitude space. The distance modulus 

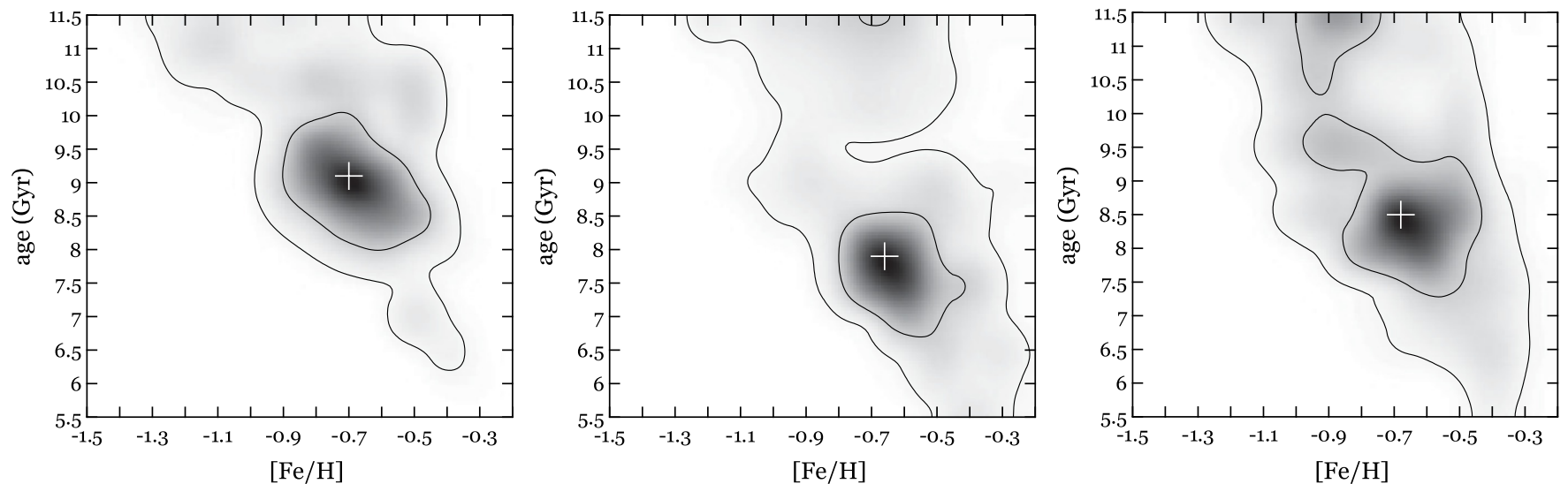

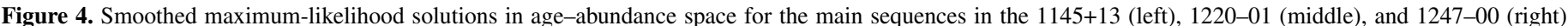

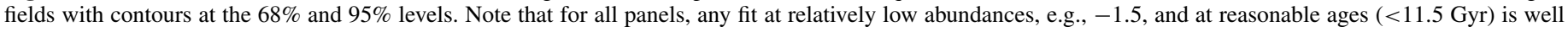
outside the $2 \sigma$ boundary.

Table 3

Properties of the Three Overdensities

\begin{tabular}{lcccc}
\hline \hline Field & $\begin{array}{c}m-M \\
(\mathrm{mag})\end{array}$ & $\begin{array}{c}\langle D\rangle_{\odot} \\
(\mathrm{kpc})\end{array}$ & $\begin{array}{c}\text { Age } \\
(\mathrm{Gyr})\end{array}$ & $\begin{array}{c}{[\mathrm{Fe} / \mathrm{H}]} \\
(\mathrm{dex})\end{array}$ \\
\hline $1145+13$ & $17.45 \pm 0.20$ & $30.9 \pm 3.0$ & $9.1_{-1.1}^{+1.0}$ & $-0.70_{-0.20}^{+0.15}$ \\
$1220-01$ & $16.93 \pm 0.21$ & $24.3 \pm 2.5$ & $7.9_{-1.2}^{+0.7}$ & $-0.66_{-0.14}^{+0.20}$ \\
$1247-00$ & $16.73 \pm 0.19$ & $22.2 \pm 2.1$ & $8.5_{-1.2}^{+1.5}$ & $-0.68_{-0.20}^{+0.24}$ \\
\hline
\end{tabular}

Note. Parameters inferred from fitting Dartmouth isochrones to the main sequence.

is sampled over a range of $15.5<m-M<18.5$ in steps of 0.025 mag. To achieve the best sensitivity in the fitting process, we used the isochrone segment between 0.5 mag brighter than the MSTO and 2 mag below.

We calculate the maximum-likelihood values $\mathcal{L}_{i}$ over a grid of isochrones covering an age range from 5.5 to $13.5 \mathrm{Gyr}$ and metallicity range $-2.5 \leqslant[\mathrm{Fe} / \mathrm{H}] \leqslant-0.2$ dex. Grid steps are $0.5 \mathrm{Gyr}$ in age, and $0.1 \mathrm{dex}$ in $[\mathrm{Fe} / \mathrm{H}]$. With a grid of $\mathcal{L}_{i}$ values, we can locate the most likely value and compute confidence intervals by interpolating between grid points. In addition to this interpolation, we smooth the likelihood values over $\sim 2$ grid points in order to provide a more conservative estimate of parameter uncertainties. In Figure 4, we present the relative density of likelihood values for the sample described above. We find that the isochrone with the highest probability has an age of $9.1 \mathrm{Gyr}$ and $[\mathrm{Fe} / \mathrm{H}]=-0.70$, with $68 \%$ and $95 \%$ confidence contours presented in the figure. The marginalized uncertainties (Table 3) about this most probable location correspond to an age of $9.1_{-1.1}^{+1.0} \mathrm{Gyr}$, a metallicity of $[\mathrm{Fe} / \mathrm{H}]=-0.70_{-0.20}^{+0.15} \mathrm{dex}$, and a distance modulus of $\mathrm{DM}=17.45 \pm 0.20 \mathrm{mag}(d=$ $30.9 \pm 3.0 \mathrm{kpc})$. They account for the varying photometric errors in the critical part $20<g<23$ of the CMD, the differences in Dartmouth isochrone shapes between 5 and $12 \mathrm{Gyr}$, and isochrone model variations. Other sources of uncertainties such as the systematic errors inherent in the process of isochrone fitting, small errors in the photometric zero points, and, at a more fundamental level, the underlying stellar physics and stellar populations used to calibrate the theoretical isochrones mean that the error bars are in general underestimates. Nevertheless, the conclusions we draw from the results are unaffected.
The best-fitting isochrone is overplotted as a blue line in the left panel of Figure 3. The associated heliocentric distance of the MS of $30.9 \pm 3.0 \mathrm{kpc}$ is in excellent agreement with the estimated Sgr Stream distance of $29 \mathrm{kpc}$ at that location (Belokurov et al. 2006). To illustrate that our results are independent of the chosen set of theoretical models, we also present the Padova isochrone (Girardi et al. 2004) with the same age, $[\mathrm{Fe} / \mathrm{H}]$, and distance (red curve). The slightly brighter turnoff and a slightly bluer lower giant branch correspond to a metallicity difference of $[\mathrm{Fe} / \mathrm{H}]_{D}-[\mathrm{Fe} / \mathrm{H}]_{P}=0.07$ dex and an age difference of $0.7 \mathrm{Gyr}$, well within the listed uncertainties derived from the Dartmouth isochrones. For a detailed comparison of the Dartmouth and Padova isochrones, we refer to Dotter et al. (2007, 2008), but we note that the differences for low-mass stars are because of differences in the treatment of the equation of state and surface boundary conditions. These differences are additional reasons why isochrone fitting was restricted to the brighter parts of the MS.

There is not much information currently available in the literature about the age, metallicity, and color of the leading tidal tail MSTO in this part of the Sgr Stream. Carlin et al. (2012) presented the most recent results for fields in the trailing tidal tail. Their Figure 21 shows the spectroscopic metallicity distribution functions (MDFs) of four fields along the regime $75^{\circ}<\Lambda_{\odot}<130^{\circ}$ with no significant metallicity gradient observed within the uncertainties. From the numbers in their Table 6 , we calculate a mean $[\mathrm{Fe} / \mathrm{H}]$ of -1.15 and a mean $\sigma_{[\mathrm{Fe} / \mathrm{H}]}$ of 0.64 . That $1 \sigma$ range of $-1.77<[\mathrm{Fe} / \mathrm{H}]<-0.49$ is statistically in agreement with our quoted $1 \sigma$ range of $-0.90<[\mathrm{Fe} / \mathrm{H}]<-0.55$.

\subsection{The VSS/VOD Fields}

The detection algorithm used by Walsh et al. (2009) to search for new ultra-faint Milky Way dwarf satellites flagged two positions, $1220-01$ and $1247-00$ (see Table 1), both located in the vicinity of two fields studied by Martínez-Delgado et al. (2007) and in the general direction of the VVS/VOD as defined by excesses of RRL stars (Vivas et al. 2001; Vivas 2002; Vivas \& Zinn 2003; Zinn et al. 2004; Ivezić et al. 2005) and of F-type MS stars (Newberg et al. 2002) spanning an R.A./decl. range of $175^{\circ}<\alpha<200^{\circ}$ and $-2.3<\delta<0.0$. The reddening-corrected CMDs of $1220-01$ and $1247-00$ are shown in Figure 2. MSs are clearly visible in the field-subtracted 
Table 4

Star Counts for the Three Main Sequences

\begin{tabular}{lcc}
\hline \hline Field & Counts & Strength \\
\hline $1145+13$ & 249 & $100 \%$ \\
$1220-01$ & 134 & $54 \% \pm 7 \%$ \\
$1247-00$ & 132 & $53 \% \pm 7 \%$
\end{tabular}

Notes. Star counts within $|g-r|<0.2$ mag of the bestfitting isochrone and 3 mag down the MS from the turnoff. The third column gives the population strength relative to the main sequence in Sgr Stream Branch A.

CMD from $g \sim 20$ down to $24.0 \mathrm{mag}$, almost as conspicuous as in our Sgr Stream field. Following the same procedure as described previously, we established the best-fitting isochrones. They are shown in Figure 3 with the corresponding maximum-likelihood solutions in Figure 4. We derive similar ages of $7.9_{-1.2}^{+0.7} \mathrm{Gyr}$ and $8.5_{-1.2}^{+1.5} \mathrm{Gyr}$ and identical metallicities of $[\mathrm{Fe} / \mathrm{H}]=-0.66_{-0.14}^{+0.20}$ and $-0.68_{-0.20}^{+0.24}$, respectively. The associated heliocentric distances are $24.3 \pm 2.5 \mathrm{kpc}$ and $22.2 \pm 2.1$ (see Table 3).

We estimate the strength of the MS population in the two VOD fields from star counts within $|g-r|<0.2$ mag of the bestfitting isochrone and 3 mag down the MS from the turnoff. These numbers are listed in Table 4 together with the corresponding percentages normalized to the 249 stars found in the $1145+13$ Sgr Stream field. The quoted uncertainties are the $\sqrt{n}$ standard error. Although the VOD fields are $\approx 13^{\circ}$ away from the main ridge of the Sgr Tidal Stream, we observe only a moderate drop in MS star counts to about $50 \%$.

Chou et al. (2010) pointed out that the lack of metal-rich RRL stars could mean that the entire VSS/VOD stellar population is metal-poor. With this point as a base, we investigate the possible presence of an old, metal-poor stellar population in the 1220-01 and 1247-00 CMDs. For that purpose, we take the estimates of -1.86 to -2.0 for the metallicity of VSS/VOD RRL stars (Duffau et al. 2006; Prior et al. 2009a; An et al. 2009), adopt an age of 11.2 Gyr and distance of $19 \mathrm{kpc}$ (Duffau et al. 2006; Newberg et al. 2007; Bell et al. 2008; Prior et al. 2009a), and overplot in the CMDs the corresponding Dartmouth and Padova isochrones (Figure 5). In both CMDs, the MSTO region of the old, metal-poor isochrones seems to match a small group of stars at $g \approx 19.7$. However, the bright MS stars that would go with this feature are mostly absent for about $0.7-1.0$ mag below the turnoff until the two isochrones merge with the best-fitting isochrones. The lack of such bright MS stars in the interval $20<g<20.8$ that could be associated with the VSS/VOD suggests that the closer population, i.e., at $19 \mathrm{kpc}$, is small at best when compared to the slightly more distant MS stars at $23 \mathrm{kpc}$.

To quantitatively assess the possibility that some of the stars above the MSTO $(g<20.2$ in Figure 3$)$ are from a smaller VOD stellar population located a few kpc closer along the line of sight, we count stars around the MSTO with colors $0.2<g-r<0.5$ in the decontaminated CMDs. The number of stars within 1 mag above and below the MSTO is listed in Table 5. The 1220-01 and 1247-00 fields have marginally more stars $(50 \% \pm 15 \%)$ above the turnoff when compared to the $1145+13$ field $(34 \% \pm 9 \%)$. If approximately one-third of the stars above the MSTO are associated with the Milky Way (i.e., leftover stars from the decontamination process), then the
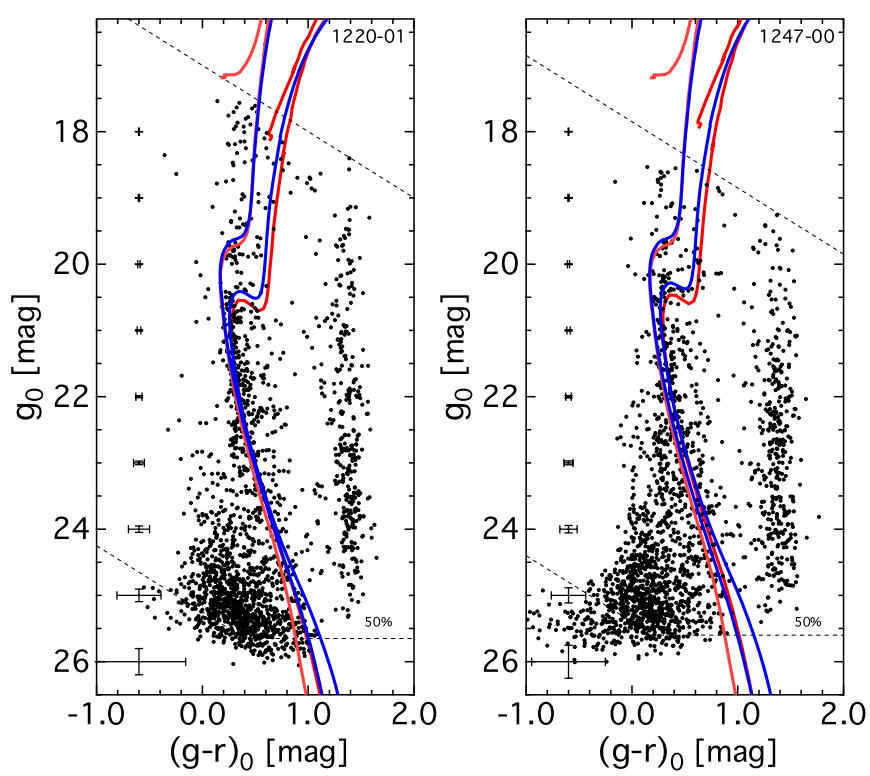

Figure 5. Same CMDs of the 1220-01 and 1247-00 fields as in Figure 3. The Dartmouth and Padova isochrones of an old (age $=11.2 \mathrm{Gyr})$, metal-poor $([\mathrm{Fe} / \mathrm{H}]=-1.9)$ stellar population at $19 \mathrm{kpc}$ as prescribed by the RRL stars in the VSS/VOD region are superimposed on the CMDs shown in Figure 3 as blue and red lines, respectively. In both fields, the MSTO region matches a small group of stars at $g \approx 19.7$. However, VSS/VOD-associated main-sequence stars are mostly absent for about $0.7-1$ mag below the turnoff.

Table 5

Number Statistics of Stars around the MSTO

\begin{tabular}{lccc}
\hline \hline Field & Above & Below & A/B Ratio \\
\hline $1145+13$ & 33 & 97 & $34 \% \pm 9 \%$ \\
$1220-01$ & 38 & 82 & $46 \% \pm 13 \%$ \\
$1247-00$ & 33 & 60 & $55 \% \pm 17 \%$ \\
\hline
\end{tabular}

Notes. Stars are counted in the $0.2<g-r<0.5$ color interval and within $1 \mathrm{mag}$ above and below the MSTO. The fourth column gives the number ratios with $\sqrt{n}$ uncertainties.

metal-poor, old VOD population is about $20 \%-30 \%$ compared to the VOD MS stars in that part of the sky.

We now discuss possible interpretations of the results from the CMD analysis.

\section{ARE THE VOD MAIN-SEQUENCE STARS PART OF THE Sgr TIDAL STREAM?}

Intriguingly, the statistically robust results, based on about 260 MS stars $(0<g-r<1, g<24)$ in the two VOD fields, are in excellent agreement with the age and metallicity derived for the MS stars in the $1145+13$ field selected to be in the direction of the highest star density of the Sgr Branch A leading arm. This immediately raises the question: are the detected VOD MS stars part of the Sgr Tidal Stream? We find that the VOD results are in good agreement with the age for the main population in the Sgr dwarf (8.0 \pm 1.5 Gyr; Bellazzini et al. 2006) and the peak in the $\mathrm{MDF}$ at $[\mathrm{Fe} / \mathrm{H}]=-0.7$ dex for the $2-3 \mathrm{Gyr}$ old $\mathrm{M}$ giants in the Sgr north leading arm in the $\Lambda_{\odot}=260^{\circ}$ region (Chou et al. 2007). We recognize that the $M$ giants might be biased to higher metallicities compared to a complete red giant branch sample, but the Bellazzini et al. study is principally based on $\mathrm{K}$ giants and does not indicate any major difference with the M-giant results. We also note that the metallicity of the VOD MS stars is 
slightly lower when compared to the stars in the Sgr core where the MDF has a wide spread from -1.0 to super-solar +0.2 and peaks at $[\mathrm{Fe} / \mathrm{H}]=-0.4 \mathrm{dex}$ (Bellazzini et al. 2008). However, this difference can be explained with the reported abundance gradient along the tidal arms (Keller et al. 2010).

Consequently, based solely on the similarity between the MS population parameters derived for our VSS/VOD fields and those observed for the Sgr dwarf and the Sgr Tidal Stream stars, it would be possible to draw the conclusion that the stellar population of the complex overdense region around $\alpha=12.5 \mathrm{~h}, \delta=0 \mathrm{deg}$ in the Virgo constellation is dominated by Sgr Tidal Stream MS stars. However, such a conclusion ignores the additional information from models of the Sgr/Galaxy interaction, which provide further constraints. These will be discussed in the next section. We note, however, that, as discussed in Section 3.2, regardless of the origin of the dominant population, our data provide little evidence for the presence of a significant separate old and metal-poor population of stars at a distance of $\sim 19 \mathrm{kpc}$.

Finally, we point out that the RRLs likely associated with Sgr in this region do not pose a problem. Abundances for 14 RRLs where the association with $\mathrm{Sgr}$ is unambiguous (e.g., Vivas et al. 2005) are low $(\langle[\mathrm{Fe} / \mathrm{H}]\rangle=-1.76$ with $\sigma=0.22)$. Moreover, the six Starkenburg et al. (2009) Group 1 stars, also likely Sgr objects, lie in this same part of the sky and have a similar mean abundance. This abundance is consistent with the RRLs in the VSS/VOD region (whether or not they are VSS objects). For instance, the two large negative $V_{\mathrm{GSR}}$ RRLs in Prior et al. (2009b), which are likely Sgr objects, have abundances $\langle[\mathrm{Fe} / \mathrm{H}]\rangle=-1.57$ similar to the Sgr RRLs of Prior et al. (2009a) and those in the Vivas et al. paper. In other words, we do not deny that there is a metal-poor Sgr population, only that it is not dominant and that the mean abundance of Sgr RRL stars is a poor indicator of the mean abundance of the Sgr Stream, as the RRLs are likely older than the bulk of the Sgr stellar population.

\section{ARE THE VOD MAIN-SEQUENCE STARS FROM A DIFFERENT HALO STRUCTURE?}

From the analysis of the deep CMDs we also gained information about the 3D location of significant populations of MS stars in three well-defined directions. These geometrical anchor points allow a comparison with the currently best Sgr Tidal Stream model by Law \& Majewski (2010, hereafter LM10). The $N$-body simulation from Law \& Majewski is based on a complete all-sky view of the Sgr Stream. It is empirically calibrated with SDSS A-branch and 2MASS M-giant stars and predicts the heliocentric distances and radial velocities of 100,000 particles stripped from the Sgr dwarf up to four orbits (approximately 3 Gyr ago).

In Figure 6, we plot heliocentric distance data as a function of orbital longitude for the simulated Sgr satellite debris from the best-fit triaxial model ( $a: b: c=1: 0.99: 0.72)$ within the assumptions of the simulation. ${ }^{16}$ The locations of the MS stars in our three fields are shown as filled circles. To illustrate the model predictions we highlight those Sgr Stream model particles that fall within half a degree of each field's orbital latitude. The chosen width equals the observed FoV. The distance distribution of the model particles is bimodal in all three cases with a minor concentration at $9 \mathrm{kpc}$ and a slightly larger population between $46<D<54 \mathrm{kpc}$ and $42<D<53 \mathrm{kpc}$, respectively.
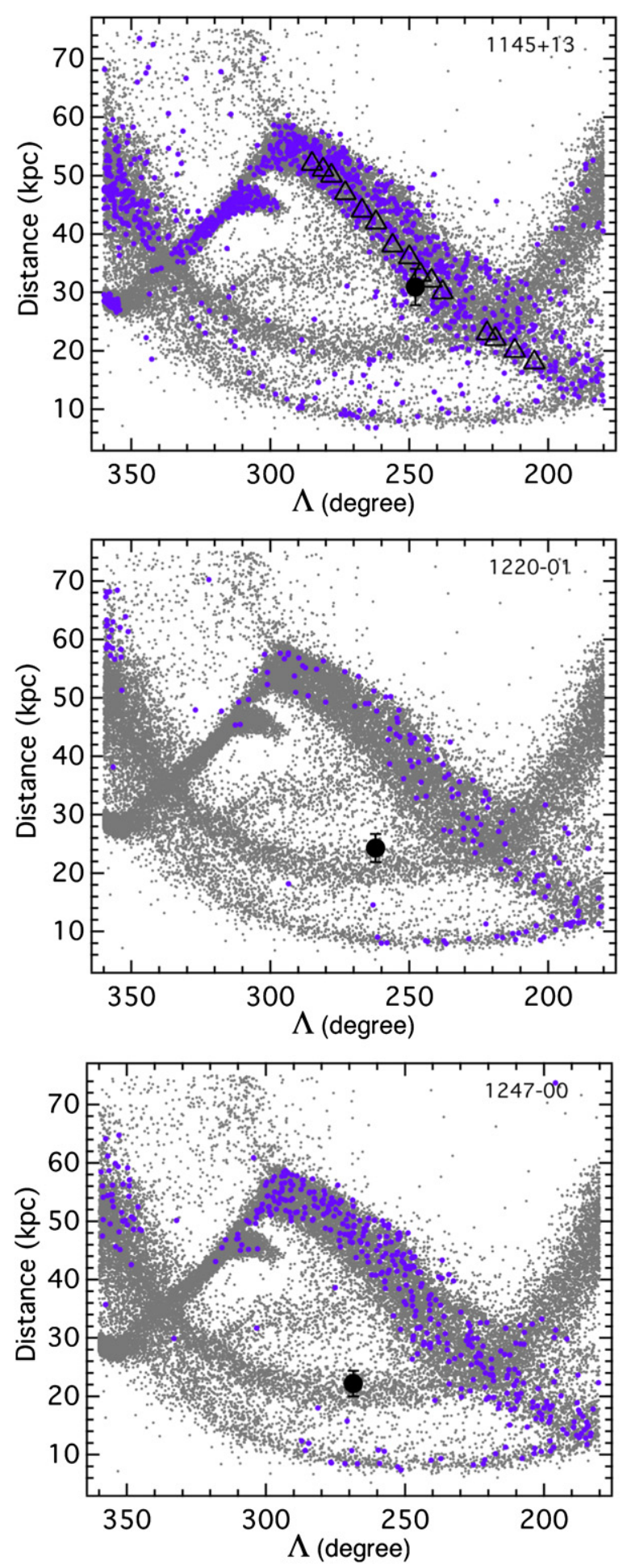

Figure 6. Distribution of the LM10 model particles in the $180^{\circ}<\Lambda_{\odot}<360^{\circ}$ range with the points in the orbital latitude interval $4.95<B_{\odot}<5.45$ (top), $15.05<B_{\odot}<15.55$ (center), and $11.15<B_{\odot}<11.65$ (bottom) highlighted in purple. The triaxial model agrees well with the spatial location of the MS stars in the Sgr Tidal Stream field 1145+13 (filled circle) and the SDSS A-stream data (triangles) from Belokurov et al. (2006). For the two VOD fields the model predicts a small concentration of Sgr Tidal Stream stars at $9 \mathrm{kpc}$ and in the distance intervals $46<D<54 \mathrm{kpc}$ and $42<D<53 \mathrm{kpc}$, respectively, but no particles at $23 \mathrm{kpc}$ where the most prominent population of main-sequence stars is detected. From particle number counts, we estimate that the Sgr Tidal Stream stars in the background should be more abundant in the 1247-00 field than in the 1220-01 field by approximately a factor of 4.3 .

\footnotetext{
16 http://www.astro.virginia.edu/ srm $4 \mathrm{n} / \mathrm{Sgr} /$ data.html
} 
The model prediction agrees well with the distance we obtained from isochrone fitting for the MS stars in the 1145+13 field (top panel of Figure 6). The good match was in fact anticipated as the LM10 Sgr Tidal Stream model was calibrated with the SDSS A-branch data from Belokurov et al. (2006; triangles in Figure 6). However, no agreement is found between the model and the VOD MS stars (filled circle in the center and bottom panels of Figure 6). No Sgr Tidal Stream stars are predicted at the distance of $23 \mathrm{kpc}$ where the maximum-likelihood fit located the MS in the 1220-01 and 1247-00 fields. If we adopt the LM10 triaxial model as the true representation of the Sgr Tidal Stream in this part of the sky, then the necessary conclusion would be that the observed MS stars at $23 \mathrm{kpc}$ originate from a different stellar structure in the Milky Way halo.

Do we find traces of Sgr Stream stars in our CMDs? To guide the eye we superimpose a Dartmouth isochrone with a metallicity of $[\mathrm{Fe} / \mathrm{H}]=-0.70$ and an age of $8 \mathrm{Gyr}$ at distances of 9 and $47 \mathrm{kpc}$ over the 1220-01 and 1247-00 CMDs in the panels of Figure 7. Looking at the course of the isochrone in both panels on the left, we note a diagonal ensemble of stars following the isochrone and running in parallel to the MS at $23 \mathrm{kpc}$ from $g_{0}=18.5 \mathrm{mag}$ down to about $g_{0}=22.5 \mathrm{mag}$. It is conceivable that these stars are indeed members of the small population of Sgr Stream stars at $9 \mathrm{kpc}$ as predicted by the model. To enhance the signal we combine the CMDs of the two fields in Figure 8. Now we can see a distinct narrow feature $\left(20.5<g_{0}<22.5,0.4<(g-r)_{0}<0.8\right)$ parallel and close to the isochrone at a distance of approximately $10 \mathrm{kpc}$. In terms of distance, small distance spread, and population size, this filamentary structure is suggestive of the $9 \mathrm{kpc} \mathrm{Sgr} \mathrm{Tidal}$ Stream predicted by the LM10 model. We note that this view offers an interesting and alternative interpretation of the origin of stars brighter than the $23 \mathrm{kpc}$ MSTO as it has been discussed in Section 4. They are MS stars of the Sgr Stream at $9 \mathrm{kpc}$ rather than the MSTO stars of an old, metal-poor population at $19 \mathrm{kpc}$.

Moving on to the $47 \mathrm{kpc}$ feature (righthand panels in Figure 7) reveals a difference between the 1220-01 and 1247-00 CMDs. In the latter field we observe more stars scattered around the isochrone while the fewer stars in the 1220-01 CMD close to the isochrone lie systematically above the line. This finding is again consistent with the picture we get from the LM10 model (see Figure 6) that predicts a population of Sgr Stream stars in the 1247-00 direction larger by a factor of 4.3. Hence it is conceivable that this excess of stars are indeed representatives of the Sgr Stream spread over $\approx 11 \mathrm{kpc}$ $(\Delta m \approx 0.5)$ from $42-53 \mathrm{kpc}$ along the line-of-sight. The bottom panel in Figure 8 shows the scatter around the isochrone in the combined CMD. We attempted to fit the data with a second population but the dominance of the $23 \mathrm{kpc}$ population and the dispersed distribution of stars produced only numerically unstable solutions. Additional data obtained over a larger field may, however, allow further validation of the Sgr Tidal Stream model and thus its use in interpreting our observations. In particular, Figure 6 suggests that in the direction of the 1247-00 field, the Sgr Stream stars from the distant (42-53 kpc) wrap are relatively frequent. Observations in this vicinity with wider area coverage may permit a stronger identification of the predicted Sgr population than is possible with the current single field.

\section{SUMMARY AND CONCLUSIONS}

We report the detection of a prominent population of MS stars in two Subaru Suprime-Cam fields 1220-01 and 1247-00 located in the overlapping region of the VSS and the VOD. The
MS stars are at a heliocentric distance of $23.3 \pm 1.6 \mathrm{kpc}$ and have an age of $8.2_{-0.9}^{+0.8} \mathrm{Gyr}$ and a mean metallicity of $[\mathrm{Fe} / \mathrm{H}]=$ $-0.67_{-0.12}^{+0.16}$ dex as estimates from the best-fitting isochrones. These parameters are similar to the age and metallicity we derived for the main stellar feature in our third Suprime-Cam field, 1145+13, centered on the leading arm of the Sgr Stream, Branch A of the bifurcation. The Sgr Stream population has a MSTO color of $(g-r)_{0} \sim 0.25$ and can be traced as faint as $g \approx 25 \mathrm{mag}$ in the CMD. From isochrone fitting we infer an age of $9.1_{-1.1}^{+1.0} \mathrm{Gyr}$ and a mean metallicity of $[\mathrm{Fe} / \mathrm{H}]=$ $-0.70_{-0.20}^{+0.15}$ dex. We believe they are the first measurements of these quantities for the leading tidal tail in this part of the Sgr Stream. The associated heliocentric distance of $30.9 \pm$ $3.0 \mathrm{kpc}$ agrees well with the estimated distance to the Sgr Tidal Stream in that direction of the Galactic halo as inferred from upper MS and turnoff stars from SDSS (Belokurov et al. 2006).

The derived parameters for the MS stars in the two VSS/VOD fields are also a good match to the age of the dominant stellar population (Pop A) in the Sgr dwarf galaxy (8.0 $\pm 1.5 \mathrm{Gyr}$; Bellazzini et al. 2006) as well as to the peak in the MDF, $[\mathrm{Fe} / \mathrm{H}]=-0.7 \mathrm{dex}$, of the 2-3 Gyr old $\mathrm{M}$ giants in the Sgr north leading arm at the orbital longitude $\Lambda_{\odot}=260^{\circ}$ (Chou et al. 2007).

The agreement in metallicity may be taken as supporting evidence that the detected VOD MS stars are from the Sgr Tidal Stream, in line with the Martínez-Delgado et al. (2007) proposition (see also Prior et al. 2009a) that the Sgr debris is a major contributor to the overdensity in Virgo. Although the measured metallicity in the VSS/VOD fields $([\mathrm{Fe} / \mathrm{H}]=$ $\left.-0.67_{-0.12}^{+0.16} \mathrm{dex}\right)$ is slightly more metal poor than the stars in the Sgr core where the MDF has a spread from -1.0 to super-solar +0.2 and peaks at $[\mathrm{Fe} / \mathrm{H}]=-0.4 \mathrm{dex}$ (Bellazzini et al. 2008), the difference is consistent with the abundance gradient along the tidal arms reported by Keller et al. (2010).

However, it must be recalled that metallicity provides only a poor constraint as other Milky Way dwarf satellites massive enough to leave a prominent stellar feature in the MW halo can be expected to have similar $[\mathrm{Fe} / \mathrm{H}]$ (Kirby et al. 2008; Meisner et al. 2012). To test the hypothesis that the VOD is dominated by stars from a different halo structure, we compared the CMDs of the VSS/VOD fields with the Sgr Tidal Stream model by Law \& Majewski (2010) based on a triaxial Galactic halo shape that is empirically calibrated with SDSS Sgr A-branch and 2MASS M-giant stars. In the surveyed directions the model makes precise predictions about the location and strength of different wraps of the Sgr Tidal Stream: a small number of Sgr Tidal Stream stars at a distance of $\approx 9 \mathrm{kpc}$ and a slightly larger population dispersed over the distance range $42<D<53 \mathrm{kpc}$ (Figure 6). Overplotted isochrones on the CMDs at the prescribed distances (Figures 7 and 8) indeed align with features that can be interpreted as the predicted Sgr Tidal Stream stars. The predicted number variation of the Sgr Tidal Stream stars between the two fields was also observed providing additional confidence in the Sgr Tidal Stream model. A more quantitative analysis of these features by means of isochrone fitting turned out to be unsuccessful because of the low contrast. A systematic, deep photometric study over a larger area will be necessary to collect more stars for further insight.

The major finding from the analysis of the CMDs of the two selected fields in the complex VSS/VOD region is a prominent MS population at $23 \mathrm{kpc}$. Given that these stars are absent in the currently best-fit $\mathrm{N}$-body simulation of the tidal disruption 

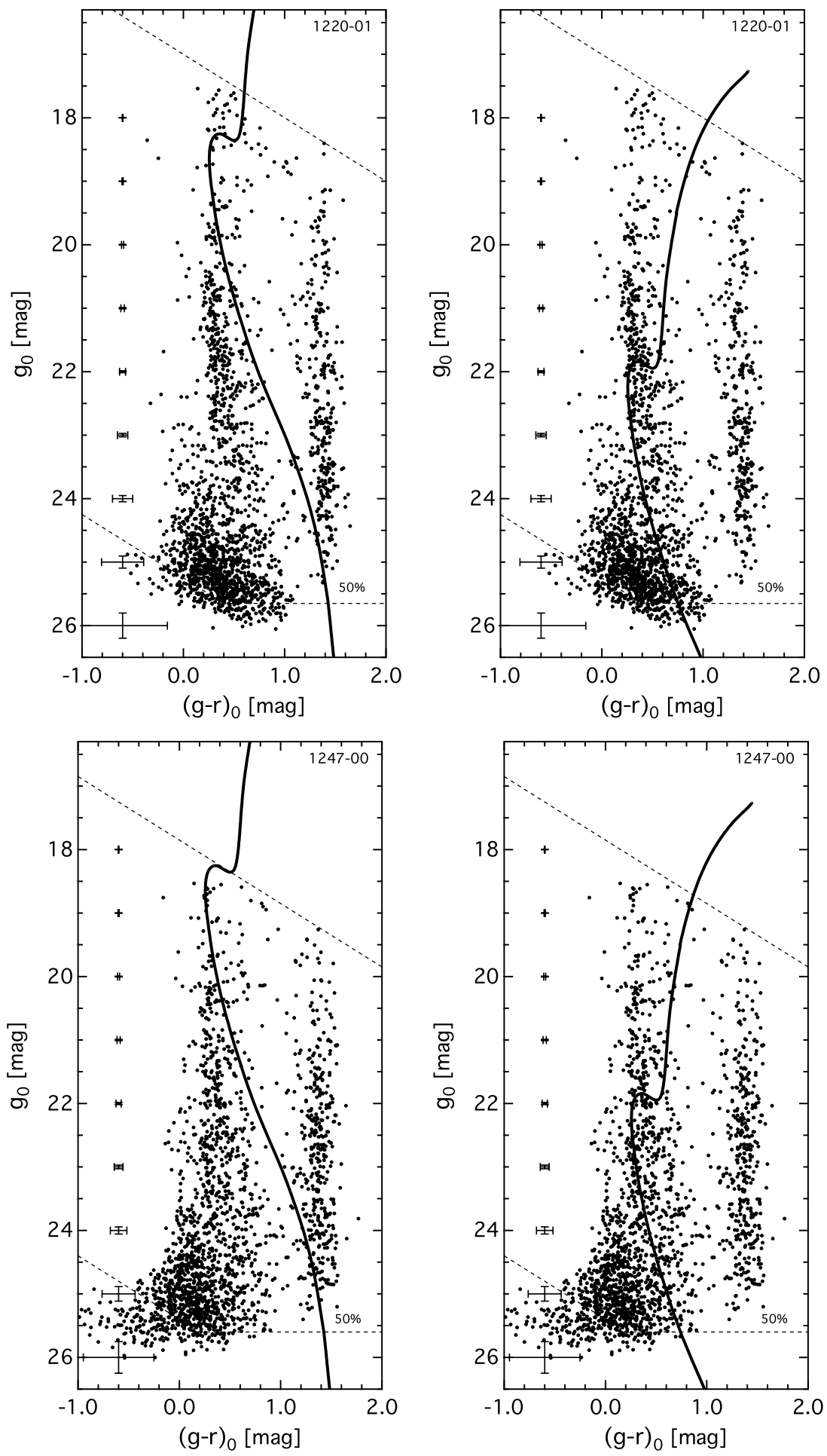

Figure 7. Dartmouth isochrones superimposed over the 1220-01 (top row) and 1247-00 CMD (bottom row) representing stellar populations at distances of 9 kpc and $47 \mathrm{kpc}$, respectively. The isochrone at $9 \mathrm{kpc}$ (left column) has a metallicity of $[\mathrm{Fe} / \mathrm{H}]=-0.70$ and an age of $8 \mathrm{Gyr}$. In both CMDs we note groups of stars that follow the isochrone resembling the small Sgr Tidal Stream population at $9 \mathrm{kpc}$ predicted by the LM10 model. This notion finds further support from the filamentous structure seen in the combined CMD (top panel, Figure 8). The panels on the right show the same isochrone at a distance of $47 \mathrm{kpc}$. Stars are scattered differently around the isochrone in the two fields, indicative for a larger population of Sgr Sream stars in the 1247-00 field, as is also predicted by the model.

of the Sgr dwarf galaxy (Law \& Majewski 2010), an association with Sgr seems unlikely. These stars appear to belong to a different stellar substructure in the Galactic halo. Additionally, we find that at the VOD location this substructure has a similar age and metallicity as the Sgr Tidal Stream stars in the leading arm, Branch A of the bifurcation. With a metallicity of
$[\mathrm{Fe} / \mathrm{H}]=-0.67$ dex the associated stars are also significantly more metal-rich than the VSS/VOD RRL stars $([\mathrm{Fe} / \mathrm{H}] \approx-1.9 \mathrm{dex})$.

The authors thank the anonymous referee for providing thoughtful comments that helped to improve the paper, and 

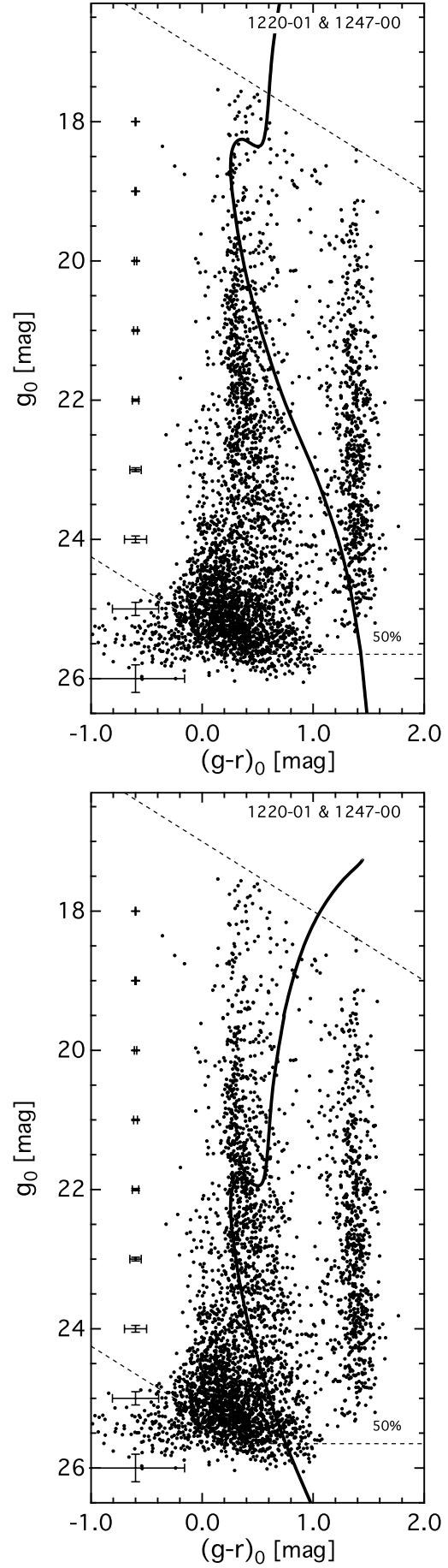

Figure 8. Same as left and center panels in Figure 7 but for the combined CMDs. Top: we note a distinct narrow feature below the isochrone at a distance of about $10 \mathrm{kpc}$ following closely the isochrone $(21.2<g<22.5,0.4<g-r<0.8)$. In terms of distance, small distance spread, and population strength, this feature could well be the $9 \mathrm{kpc}$ Sgr Tidal Stream predicted by the LM10 model. Bottom: it is plausible that the stars scattered around the $47 \mathrm{kpc}$ isochrone are from the Sgr Tidal Stream that extends from $42<D<53 \mathrm{kpc}$ along the line of sight.

Renee Kraan-Korteweg for reading the early versions of the manuscript. H.J. and P.K. acknowledge financial support from the Go8-DAAD-Australia/Germany Joint Research Cooperation Scheme and H.J. and P.T. acknowledge financial support from the Access to Major Research Facilities Programme, which is a component of the International Science Linkages established under the Australian Government innovation statement, Backing Australia's Ability. This research is also supported in part by the Australian Research Council through Discovery Projects grants DP0878137 and DP120100475. B.W. thanks NSF AST 0908446. A.J. and M.Z. acknowledge support from BASAL CATA PFB-06, FONDAP CFA 15010003, the Chilean Ministry for the Economy, Development, and Tourisms Programa Iniciativa Científica Milenio through grant P07-021-F, awarded to The Milky Way Millennium Nucleus, and Fondecyt REGULAR 1110393. E.O. acknowledges American NSF support through grant AST-0807498. The authors are grateful to David Law and Steven Majewski for making the details of their model data publicly available.

\section{REFERENCES}

An, D., Johnson, J. A., Beers, et al. 2009, ApJL, 707, L64

Bell, E. F., Zucker, D. B., Belokurov, V., et al. 2008, ApJ, 680, 295

Bellazzini, M., Correnti, M., Ferraro, F. R., Monaco, L., \& Montegriffo, P. 2006, A\&A, 446, L1

Bellazzini, M., Ferraro, F. R., \& Buonanno, R. 1999, MNRAS, 307, 619

Bellazzini, M., Ibata, R. A., Chapman, S. C., et al. 2008, AJ, 136, 1147

Belokurov, V., Zucker, D. B., Evans, N. W. R., et al. 2006, ApJL, 642, L137

Bertin, E., \& Arnouts, S. 1996, A\&AS, 117, 393

Bonaca, A., Jurić, M., Ivezić, Z., et al. 2012, AJ, 143, 105

Carlin, J. L., Majewski, S. R., Casetti-Dinescu, D. I., et al. 2012, ApJ, 744, 25

Chen, B., Stoughton, C., Smith, J. A., et al. 2001, ApJ, 553, 184

Chou, M., Cunha, K., Majewski, S. R., et al. 2010, ApJ, 708, 1290

Chou, M., Majewski, S. R., Cunha, K., et al. 2007, ApJ, 670, 346

Correnti, M., Bellazzini, M., Ibata, R. A., Ferraro, F. R., \& Varghese, A. 2010, ApJ, 721, 329

Dotter, A., Chaboyer, B., Jevremović, D. J., et al. 2007, AJ, 134, 376

Dotter, A., Chaboyer, B., Jevremović, D., et al. 2008, ApJS, 178, 89

Duffau, S., Zinn, R., Vivas, A. K., et al. 2006, ApJL, 636, L97

Fadely, R., Willman, B., Geha, M., et al. 2011, AJ, 142, 88

Fellhauer, M., Belokurov, V., Evans, N. W., et al. 2006, ApJ, 651, 167

Frayn, C. M., \& Gilmore, G. F. 2002, MNRAS, 337, 445

Girardi, L., Grebel, E. K., Odenkirchen, M., \& Chiosi, C. 2004, A\&A, 422, 205

Girardi, L., Groenewegen, M. A. T., Hatziminaoglou, E., \& da Costa, L. 2005, A\&A, 436, 895

Ibata, R., Irwin, M., Lewis, G. F., \& Stolte, A. 2001, ApJL, 547, L133

Ibata, R. A., Gilmore, G., \& Irwin, M. J. 1994, Natur, 370, 194

Ivezić, Ž., Vivas, A. K., Lupton, R. H., \& Zinn, R. 2005, AJ, 129, 1096

Jurić, M., Ivezić, Ž., Brooks, A., et al. 2008, ApJ, 673, 864

Keller, S. C., da Costa, G. S., \& Prior, S. L. 2009, MNRAS, 394, 1045

Keller, S. C., Yong, D., \& Da Costa, G. S. 2010, ApJ, 720, 940

Kirby, E. N., Simon, J. D., Geha, M., Guhathakurta, P., \& Frebel, A. 2008, ApJL, $685, \mathrm{~L} 43$

Kroupa, P., Famaey, B., de Boer, K. S., et al. 2010, A\&A, 523, 32

Kunder, A., \& Chaboyer, B. 2009, AJ, 137, 4478

Law, D. R., Johnston, K. V., \& Majewski, S. R. 2005, ApJ, 619, 807

Law, D. R., \& Majewski, S. R. 2010, ApJ, 714, 229

Layden, A. C., \& Sarajedini, A. 2000, AJ, 119, 1760

Majewski, S. R., Skrutskie, M. F., Weinberg, M. D., \& Ostheimer, J. C. 2003, ApJ, 599, 1082

Martínez-Delgado, D., Aparicio, A., Gómez-Flechoso, M. Á., \& Carrera, R. 2001, ApJL, 549, L199

Martínez-Delgado, D., Gómez-Flechoso, M. Á., Aparicio, A., \& Carrera, R. 2004, ApJ, 601, 242

Martínez-Delgado, D., Peñarrubia, J., Jurić, M., Alfaro, E. J., \& Ivezić, Z. 2007, ApJ, 660, 1264

Meisner, A. M., Frebel, A., Jurić, M., \& Finkbeiner, D.P. 2012, ApJ, 753, 116

Miyazaki, S., Komiyama, Y., Sekiguchi, M., et al. 2002, PASJ, 54, 833

Monaco, L., Ferraro, F. R., Bellazzini, M., \& Pancino, E. 2002, ApJL, 578, L47

Newberg, H. J., Yanny, B., Cole, N., et al. 2007, ApJ, 668, 221

Newberg, H. J., Yanny, B., Grebel, E. K., et al. 2003, ApJL, 596, L191

Newberg, H. J., Yanny, B., Rockosi, C., et al. 2002, ApJ, 569, 245

Ouchi, M., Shimasaku, K., Okamura, S., et al. 2004, ApJ, 611, 660

Prior, S. L., Da Costa, G. S., \& Keller, S. C. 2009a, ApJ, 704, 1327

Prior, S. L., Da Costa, G. S., Keller, S. C., \& Murphy, S. J. 2009b, ApJ, 691,306

Skrutskie, M. F., Cutri, R. M., Stiening, R., et al. 2006, AJ, 131, 1163

Starkenburg, E., Helmi, A., Morrison, H. L., et al. 2009, ApJ, 698, 567 
Vanhollebeke, E., Groenewegen, M. A. T., \& Girardi, L. 2009, A\&A, 498, 95 Vivas, A. K. 2002, PhD thesis, Yale Univ.

Vivas, A. K., Jaffé, Y. L., Zinn, R., et al. 2008, AJ, 136, 1645

Vivas, A. K., \& Zinn, R. 2003, MmSAI, 74, 928

Vivas, A. K., Zinn, R., Andrews, P., et al. 2001, ApJL, 554, L33

Vivas, A. K., Zinn, R., \& Gallart, C. 2005, AJ, 129, 189
Walsh, S. M., Willman, B., \& Jerjen, H. 2009, AJ, 137, 450

Yagi, M., Kashikawa, N., Sekiguchi, M., et al. 2002, AJ, 123, 66

York, D. G., Adelman, J., Anderson, J. E., Jr., et al. 2000, AJ, 120, 1579

Zinn, R., Vivas, A. K., Gallart, C., \& Winnick, R. 2004, in ASP Conf. Ser. 327, Satellites and Tidal Streams, ed. F. Prada, D. Martinez Delgado, \& T. J. Mahoney (San Francisco, CA: ASP), 92 